\title{
Effects of nanoparticle size on antitumor activity of I0-hydroxycamptothecin-conjugated gold nanoparticles: in vitro and in vivo studies
}

This article was published in the following Dove Press journal:

International Journal of Nanomedicine

7 March 2016

Number of times this article has been viewed

\author{
Hanmei Bao ${ }^{1,2}$ \\ Qing Zhang ${ }^{2,3}$ \\ Hui Xu',2 \\ Zhao Yan ${ }^{1,2}$
}

'Department of Clinical

Pharmacology, ${ }^{2}$ Key Laboratory of

Cancer Prevention and Therapy,

National Clinical Research Center for

Cancer, ${ }^{3}$ Department of Hematology,

Tianjin Medical University Cancer

Institute and Hospital, Tianjin, People's

Republic of China
Correspondence: Zhao Yan Department of Clinical Pharmacology, Tianjin Medical University Cancer Institute and Hospital, Huan-Hu-Xi Road, Ti-Yuan-Bei, Hexi District, Tianjin 300060, People's Republic of China Tel +86 2223340123 (Ext 60I2) Fax +86222352 4155

Email zhao.yan0219@hotmail.com
Abstract: Gold nanoparticles (AuNPs) have emerged as a promising anticancer drug delivery scaffold. However, some controversial points still require further investigation before clinical use. A complete understanding of how animal cells interact with drug-conjugated AuNPs of well-defined sizes remains poorly understood. In this study, we prepared a series of 10-hydroxycamptothecin (HCPT)-AuNP conjugates of different sizes and compared their cytotoxic effect in vitro and antitumor effect in vivo. Transmission electron micrographs showed that the NPs had a round, regular shape with a mean diameter of $\sim 10,25$, and $50 \mathrm{~nm}$. An in vitro drug release study showed that HCPT was continuously released for 120 hours. HCPT-AuNPs showed greater cytotoxic effects on the MDA-MB-231 cell line compared with an equal dose of free HCPT. Notably, HCPT-AuNPs of an average diameter of $50 \mathrm{~nm}$ (HCPTAuNPs-50) had the greatest effect. Furthermore, administration of HCPT-AuNPs-50 showed the most tumor-suppressing activity against MDA-MB-231 tumor in mice among all treatment groups. The results indicate that AuNPs not only act as a carrier but also play an active role in mediating biological effects. This work gives important insights into the design of nanoscale delivery and therapeutic systems.

Keywords: drug delivery, cancer treatment, drug-containing nanocomposites, particle size

\section{Introduction}

Over the past decade, due to their good biocompatibility, ease of synthesis, monodispersity, and tunable surface functionality, ${ }^{1}$ gold nanoparticles (AuNPs) have shown excellent potential for the delivery of a wide range of antitumor substances such as oxaliplatin, ${ }^{2}$ cisplatin, ${ }^{3}$ and paclitaxel. ${ }^{4}$ Additionally, the synthesis of AuNPs can be easily controlled to obtain NPs of a wide range of sizes. ${ }^{5}$ The size of NPs has been shown to be one of the most important factors affecting biodistribution, tissue uptake, and applications in the biomedical fields. ${ }^{6}$ It has been reported that the cellular uptake of NPs ranging from 15 to $100 \mathrm{~nm}$ depends upon the particle size. ${ }^{7}$ Considerable research has recently been carried out on the biodistribution, cellular uptake, and in vivo activity of AuNPs. ${ }^{8}$ NPs of a particular size $(<200 \mathrm{~nm})$ show efficient extravasation into leaky tumor vasculature and accumulation in tumor tissues via an enhanced permeability and retention effect. ${ }^{9}$ Particle size-dependent biodistribution and toxicity of AuNPs have been widely studied in vivo. ${ }^{10}$ Generally speaking, a smaller particle size is characteristic of higher transvascular and interstitial transport. ${ }^{11,12}$ For example, $20 \mathrm{~nm}$ NPs showed significantly higher permeability in tumors compared with $125 \mathrm{~nm}$ NPs. Therefore, to be effective, a drug carrier must be sufficiently small to perfuse out of the bloodstream, penetrate the vessels, and reach the tumor site. A type of size-shrinkable 
nanocarrier-based drug delivery system was developed for the inhibition of tumor growth and metastasis, with the capacity of targeting and changing size. ${ }^{13,14}$ The prepared NPs could actively target a tumor via receptor-mediated endocytosis, followed by shrinkage from a large size to a small size due to the degradation of the NPs. Current nanomedicine is focused on drug-tethered NPs of relatively larger sizes $(>50 \mathrm{~nm})$. To the best of our knowledge, the exact effect of size on the therapeutic performance of drug-incorporated AuNPs is still not well understood. ${ }^{15}$

Camptothecin (CPT), a natural plant alkaloid extracted from Camptotheca acuminata, a plant native to China, shows significant antitumor activity against various tumors, including lung, ovarian, breast, pancreas, and stomach tumors, via the inhibition of the activity of DNA topoisomerase I. ${ }^{16}$ Due to the promising and potent antitumor activity of CPT, many CPT derivatives have been synthesized and have received increasing attention. 10-Hydroxycamptothecin (HCPT), a natural CPT analog with a 10-hydroxy substituent, has shown stronger antitumor activity and lower toxicity in both experimental animal and human trials compared with CPT. ${ }^{17}$ It has been widely used in the treatment of gastric carcinoma, hepatoma, leukemia, and tumors of the head and neck. ${ }^{18}$ However, the therapeutic potential of HCPT has been restricted by its low aqueous solubility and instability. It has been shown that the preservation of the $\alpha$-hydroxy- $\delta$-lactone is crucial for antitumor activity. ${ }^{19}$ Unfortunately, the delivery of the lactone form is quite challenging, since the lactone ring is readily opened and converted into the carboxylate form under physiological and alkaline conditions. ${ }^{20}$ Therefore, an effective drug delivery system for HCPT is important for therapeutic purposes.

Numerous novel drug delivery systems for HCPT have been previously designed to overcome its hydrophobic and unstable characteristics. HCPT has been conjugated to poly (ethylene glycol) (PEG) to improve its water solubility. However, its therapeutic efficiency in colorectal LS174 subcutaneous tumors was not improved, due to its relatively short half-life in plasma. ${ }^{21}$ Other approaches including liposomes, ${ }^{22}$ microspheres, ${ }^{23}$ submicron emulsions,${ }^{24}$ chitosan complexation, ${ }^{25}$ and the use of water- and lipid-soluble prodrugs ${ }^{26}$ have been proposed. However, the drug loading capacity, encapsulation, physical stability, and adverse side effects from the solubilization or encapsulation excipients are also in question. Furthermore, the relatively large size of the conjugate may limit its applications in tumor inhibition via the intravenous route.

Therefore, in this study, we report the synthesis of a series of HCPT-AuNPs for improved anticancer drug delivery that have tunable sizes from 10 to $50 \mathrm{~nm}$. We wanted to add our experience with relatively small NPs to the body of this research. The NPs were characterized using transmission electron microscopy (TEM), ultraviolet-visible (UV-vis) absorption, and dynamic light scattering (DLS). We then evaluated the size-dependent uptake and cytotoxic activity of the NPs in breast cancer cells. We also investigated the antitumor effect and the tolerance to 10-50 nm HCPT-AuNP conjugates in mouse tumor xenografts.

\section{Materials and methods Materials}

Tetrachloroauric acid $\left(\mathrm{HAuCl}_{4} \cdot 3 \mathrm{H}_{2} \mathrm{O}\right)$, sodium borohydride $\left(\mathrm{NaBH}_{4}\right)$, and sodium citrate were bought from Jiangtian Chemical Engineering Co., Ltd. (Tianjin, People's Republic of China). HCPT and $\alpha$-methoxy-poly (ethylene glycol)- $\omega$ mercapto (PSH; Mw 5,000 Da) were purchased from Sigma (St Louis, MO, USA). Luciferase-expressing MDA-MB-231 cells (MDA-MB-231-luc) were a kind gift from Professor Niu Ruifang (Tianjin Medical University Cancer Institute and Hospital). The reagent 3-(4,5-dimethylthiazol-2-yl)2,5-diphenyltetrazolium bromide (MTT) and Dulbecco's Modified Eagle's Medium (DMEM) were purchased from Solarbio Science \& Technology Co., Ltd (Beijing, People's Republic of China). Fetal bovine serum (FBS) was purchased from Gibco (Waltham, MA, USA).

\section{Preparation and characterization of NPs Synthesis of AuNPs}

We synthesized a series of AuNPs of diameters of 6.4, 17.6, and $38.2 \mathrm{~nm}$ as measured by TEM with a JEM-100CVII instrument (JEOL, Tokyo, Japan). Synthesis of the AuNPs was accomplished by the reduction of $\mathrm{HAuCl}_{4}\left(\mathrm{Au}^{3+}\right)$ to neutral gold $\left(\mathrm{Au}^{0}\right)$ with sodium citrate. ${ }^{27}$ Briefly, $5.0 \mathrm{~mL}$ of $0.1 \% \mathrm{HAuCl}_{4} \cdot 3 \mathrm{H}_{2} \mathrm{O}$ solution was added into $50 \mathrm{~mL}$ of double-distilled water and heated under reflux until boiling. Then, various volumes of $1 \%$ sodium citrate were added with vigorous stirring. Boiling continued for 15 minutes. By varying the sodium citrate-to- $\mathrm{HAuCl}_{4}$ concentration ratio, particles of sizes of 17.6 and $38.2 \mathrm{~nm}$ were obtained. An aqueous solution of $\mathrm{NaBH}_{4}$ and sodium citrate was added to the $\mathrm{HAuCl}_{4} \cdot 3 \mathrm{H}_{2} \mathrm{O}$ solution with vigorous stirring to obtain AuNPs with diameter of $6.4 \mathrm{~nm}$.

\section{Synthesis of AuNPs containing PEG-backbones (PSH-AuNPs)}

Gold nano-conjugates (PSH-AuNPs) were prepared using a single-step incubation by adding an excess of PSH to an AuNPs solution at room temperature with magnetic stirring for 2 hours. The particles were then washed three times 
by ultra-centrifugation at $14,000 \mathrm{rpm}$ for 20 minutes. The resulting nano-conjugates were collected, lyophilized, and stored at $4^{\circ} \mathrm{C}$ until use.

\section{Synthesis of HCPT-conjugated NPs (HCPT-AuNPs)}

To each solution of different-sized PSH-AuNPs in distilled water $(1 \mathrm{~mL}), 20 \mu \mathrm{L}$ HCPT dissolved in dimethyl sulfoxide was added, and then the solutions were incubated at room temperature with magnetic stirring overnight. Subsequently, the solution was ultra-centrifuged at 14,000 rpm for 20 minutes three times and rinsed with $0.1 \mathrm{M}$ phosphatebuffered saline (PBS) between each centrifugation. Finally, the complexes were redispersed in PBS to an appropriate volume for further study.

\section{Characterization of NPs}

UV-vis absorption spectra were recorded at room temperature using Spectra max PLUS 384 (Molecular Devices, Sunnyvale, CA, USA) in the range of 400-800 nm. NPs were placed on a copper mesh coated with an amorphous carbon film for TEM at an acceleration voltage of $100 \mathrm{kV}$ to determine particle size and morphology. The zeta-potential of the NPs was measured using DLS (ZEN 3600; Malvern, UK).

\section{Stability study}

For stability analysis, HCPT-AuNP conjugates and PSHAuNPs of different sizes were incubated at $37^{\circ} \mathrm{C}$ under the following conditions: $0.03 \mathrm{M}$ PBS at $\mathrm{pH} 7.4$; and $0.03 \mathrm{M}$ PBS at $\mathrm{pH} 5.5$, lowered by the addition of $1 \mathrm{~mol} / \mathrm{L} \mathrm{HCl}$, in the presence of FBS (at pH 7.4 in $0.03 \mathrm{M}$ PBS solution with $2 \%$ serum) and DMEM (pH 7.4 with $10 \%$ FBS). The absorbance of the nano-conjugates was recorded at $520 \mathrm{~nm}$ before incubation and at $0.5,1,1.5,2,4,6,8$, and 12 hours post-incubation. We also investigated the size variation of the nano-conjugates at predetermined time points under the conditions described.

\section{HCPT release behavior in vitro}

HCPT adsorption/conjugation onto NPs was accomplished according to the literature. ${ }^{28}$ An excess of HCPT was reconstituted in PSH-AuNPs solution and incubated at room temperature for up to 12 hours. The amount of HCPT adsorbed onto the NPs was considered to be $100 \%$ after 12 -hour incubation. The mixture was then centrifuged to separate the unadsorbed HCPT from the HCPT-AuNPs conjugate. The amount of adsorbed HCPT was calculated from the difference between the initial and the free HCPT concentrations. The HCPT release behavior in vitro was studied by incubating a solution of the HCPT-AuNPs conjugate in culture medium or PBS solution containing Tween $80(0.1 \%$, w/v), which was placed in an air bath shaker at $150 \mathrm{rpm}$ at $37^{\circ} \mathrm{C} \pm 0.5^{\circ} \mathrm{C}$. At set time intervals, samples were collected from the medium, and the same volume of fresh release medium was added. Released HCPT was separated by centrifugation, and the amount of released HCPT was determined by high-performance liquid chromatography. A reversed-phase C18 column $(4.6 \times 250 \mathrm{~mm}, 5.0 \mathrm{~mm})$ was used to separate samples $(20 \mu \mathrm{L}$ injection volume). The measurement was conducted with methanol and water $(55 / 45, \mathrm{v} / \mathrm{v})$ at a flow rate of $1.0 \mathrm{~mL} / \mathrm{min}$ and at a detection wavelength of $382 \mathrm{~nm}$.

\section{Cellular uptake of HCPT}

MDA-MB-231 human breast cancer cells $\left(2 \times 10^{5}\right)$ were cultured in DMEM supplemented with $10 \%$ FBS in a six-well plate for 24 hours, followed by treatment with HCPT-AuNPs of different sizes for 4 hours. The solution was removed, and the cells were washed with ice-cold PBS three times and lysed by freeze-thawing and ultrasonic methods. The amount of HCPT in the cell suspension was then determined by high-performance liquid chromatography as described. Ethical permission for the human breast cancer cells use was obtained from the Ethics Committee of Tianjin Medical University Cancer Institute and Hospital.

\section{In vitro cytotoxicity assay}

The cytotoxicity of NPs of different sizes was determined by the MTT assay. ${ }^{29}$ MDA-MB-231 human breast cancer cells were cultured in the presence of DMEM supplemented with $10 \% \mathrm{FBS}$, penicillin $(100 \mathrm{U} / \mathrm{mL})$, and streptomycin $(100 \mathrm{mg} / \mathrm{mL})$. The cells were seeded in 96-well plates at a density of $5 \times 10^{3}$ cells per well and were allowed to attach and grow for 2 days. The cells were exposed to PSH-AuNPs, HCPT-AuNPs, or HCPT over a range of concentrations for 48 hours, and then the medium was replaced by a drug-free medium for a further 24 hours. After incubation, $20 \mu \mathrm{L}$ of MTT solution ( $5 \mathrm{mg} / \mathrm{mL}$ in PBS) was added to each well and incubated at $37^{\circ} \mathrm{C}$ in $5 \% \mathrm{CO}_{2}$ for 4 hours. The solution in the wells was then aspirated gently, and $150 \mu \mathrm{L}$ of dimethyl sulfoxide was added to dissolve the formazan crystals. After shaking for 5 minutes, the absorbance was measured at $570 \mathrm{~nm}$ with a Spectra max PLUS 384 (Molecular Devices). The results were expressed as the mean percentage of cell viability relative to untreated cells.

\section{Animal studies}

\section{Animal care}

The experimental design of this study was approved by the Ethics Committee of Tianjin Medical University Cancer 
Institute and Hospital. All animals were used in accordance with protocols approved by the Institutional Animal Care and Use Committee of the Tianjin Medical University Cancer Institute and Hospital. Female athymic nude mice, aged 3-4 weeks, were purchased from Institute of Laboratory Animal Sciences, Peking Union Medical College (Beijing, People's Republic of China). Animals received a standard diet and water ad libitum.

\section{Antitumor effects in MDA-MB-23 I tumor-bearing mice}

MDA-MB-231 breast carcinoma cells were cultured, harvested during log-phase growth, and resuspended in $50 \%$ matrigel. Subsequently, $\sim 5 \times 10^{6}$ cells $(0.1 \mathrm{~mL}$ cell suspension) were inoculated subcutaneously into the right flank of the nude mice. The tumor take rate was $\sim 90 \%$. The animals were examined twice weekly for the development of palpable tumors at the site of injection. The tumor volume was estimated by using the formula $a b^{2} / 2$, where $a$ and $b$ represent tumor length and width, respectively. When the average tumor volume reached $200-250 \mathrm{~mm}^{3}$, the tumor-bearing mice were randomly assigned to six groups. The negative control group was treated with saline, and the experimental groups received HCPT, PSH-AuNPs, and HCPT-AuNPs (of three different sizes), at an intraperitoneal (ip) dose of $2.0 \mathrm{mg}$ $\mathrm{HCPT} / \mathrm{kg}$ once daily on days $1,5,9,13$, and 17 (q4d×5). The tumor length and width were measured using calipers, and the tumor volume was calculated. The tumor volume was converted to tumor weight assuming that $1 \mathrm{~mm}^{3}$ was equal to $1 \mathrm{mg}$ of tumor weight. The tumor volume was determined at least twice weekly for 21 days after treatment.

\section{In vivo bioluminescent imaging}

Measurement of luminescence was conducted before administration, and at 10 and 20 days post-administration using an in vivo IVIS spectrum imaging system (Caliper Life Sciences, Waltham, MA, USA). The mice were given a $150 \mathrm{mg} / \mathrm{kg}$ dose of D-luciferin by ip injection 15 minutes before imaging. General anesthesia was induced with 5\% isoflurane and continued during the procedure with $2.5 \%$ isoflurane introduced via a nose cone. The data were analyzed using Xenogen Living Image ${ }^{\circledR}$ software.

\section{Tolerance}

The mice were weighed twice every 3 days during the study and examined for overt signs of adverse side effects related to the drug, such as appetite, diarrhea, life span, and behavior. Unacceptable toxicity was defined as a loss $\geq 20 \%$ of mean body weight accompanied by death of animals in the same group. After the mice were sacrificed with an overdose of sodium pentobarbital at the end of study, the heart, liver, spleen, lung, and kidney were retrieved, cut into $5 \mu \mathrm{m}$ sections, and stained with hematoxylin and eosin, and the pathology was analyzed under a Nikon microscope (Eclipse Ti; Nikon, Tokyo, Japan).

\section{Statistical analysis}

Statistical analysis was performed using Excel 2007 and SPSS version 12.0 (SPSS Inc., Chicago, IL, USA). All values were expressed as the mean \pm standard deviation (SD). Statistical significance was calculated using the Student's $t$-test (within groups) or one-way analysis of variance (between groups). A value of $P<0.05$ was considered to be statistically significant.

\section{Results and discussion Nanoparticle characterization}

For the purposes of this study, we synthesized a series of HCPT-AuNP formulations with expected particle sizes of $\sim 10,25$, and $50 \mathrm{~nm}$ dispersed in PBS, and characterized their properties by morphology, particle size, size distribution, and surface charge. NPs of each size have a spherical morphology with quite a narrow size distribution (Figure 1A-C). The hydrodynamic sizes obtained from the three different NPs were slightly larger than that determined by TEM because of the shrinkage of the HCPT-AuNPs during TEM sample preparation. ${ }^{30}$ In other words, the DLS determined the hydrodynamic diameter or the "equivalent sphere diameter" in solution, whereas the TEM images were obtained in the absence of a solvent. ${ }^{31}$ The zeta potential measurements show that the surface potential of the NPs was negative (Table 1). The surface plasmon of resonance band of each size of NPs was examined using UV-vis spectroscopy. The HCPT-AuNPs that we prepared showed an absorption shift from 520 to $550 \mathrm{~nm}$ as the size increased (Figure 1D).

\section{Stability analyses}

To evaluate the stability of different-sized PSH-AuNPs and HCPT-AuNPs in various environments that are known to immediately induce the aggregation of unmodified AuNPs, the UV-vis absorption intensity at $520 \mathrm{~nm}$ was measured as a function of time, taking HCPT-AuNPs of an average diameter of $50 \mathrm{~nm}$ (HCPT-AuNPs-50) as an example (depicted in Figure 2A). The optical absorbance of the NPs suspension at $520 \mathrm{~nm}$ was slightly reduced for the HCPTAuNPs-50 conjugate in $0.03 \mathrm{M} \mathrm{PBS}$ at $\mathrm{pH} 7.4$. The relative 

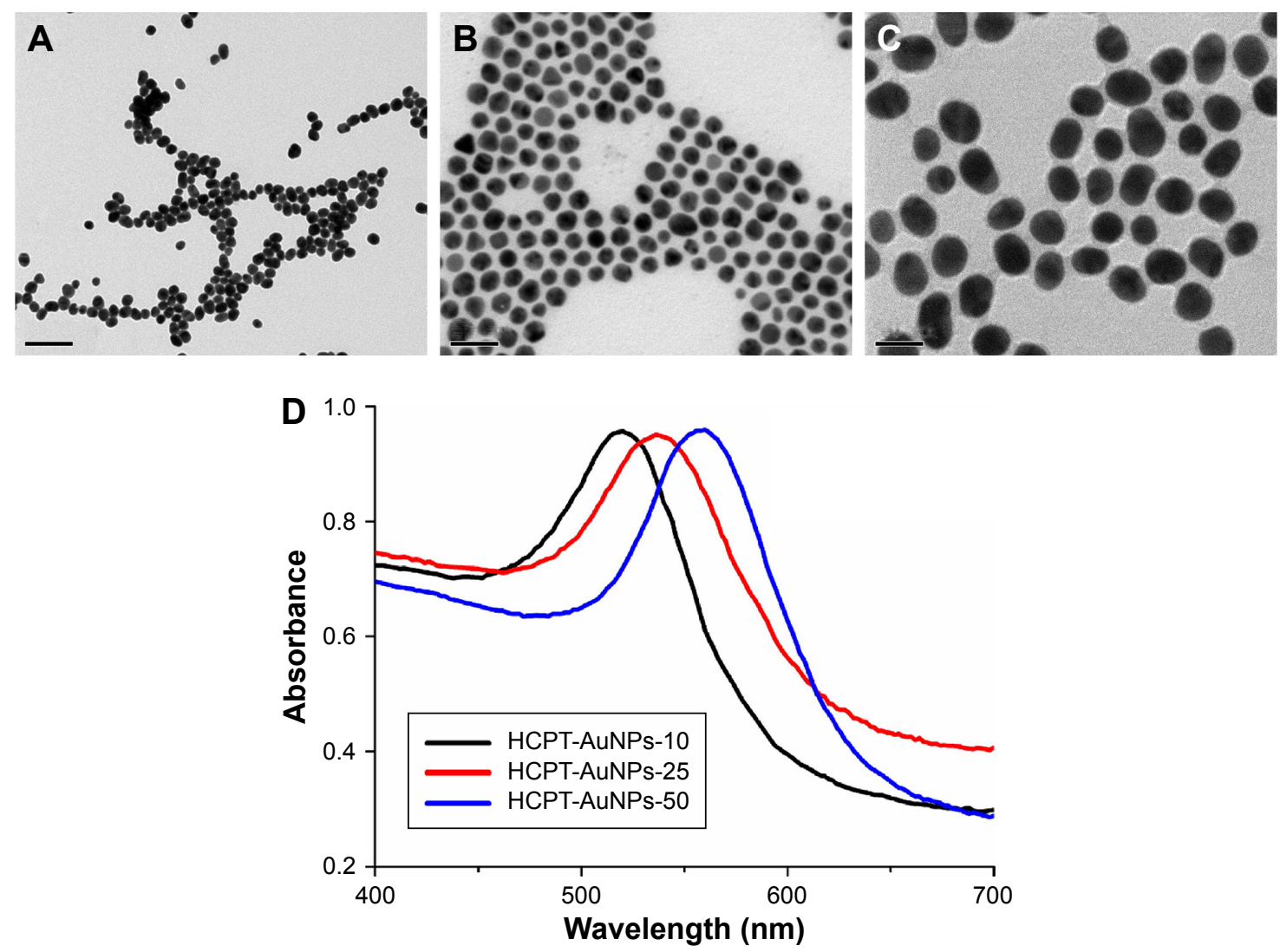

Figure I Characterization of nanoparticles.

Notes: (A-C) Transmission electron micrographs and (D) UV-vis absorption spectra of different-sized HCPT-AuNP formulations (scale bar 50 nm). HCPT-AuNPs-I0, 25 and 50: HCPT-AuNPs of an average diameter of 10,25 and $50 \mathrm{~nm}$. The magnification of the picture is $(\times 100,000)$.

Abbreviations: UV-vis, ultraviolet-visible; HCPT, I0-hydroxycamptothecin; AuNPs, gold nanoparticles.

absorbance of the groups in PBS at $\mathrm{pH} 7.4$ with and without $2 \%$ serum 12 hours post-incubation was $81.8 \% \pm 1.0 \%$, and $79.4 \% \pm 1.1 \%$, respectively. The relative absorbance of the group in cell culture media was $82.6 \% \pm 2.3 \%$. Further, the relative absorbance of the group at $\mathrm{pH} 5.5$ was $95.7 \% \pm 0.6 \%$ at the end of the incubation time. These characteristics were

Table I Physiochemical characterization of PSH-AuNPs and HCPT-AuNPs formulations (data expressed as the mean \pm $\mathrm{SD}, \mathrm{n}=3$ )

\begin{tabular}{lllll}
\hline NPs & $\begin{array}{l}\text { Diameter } \\
(\mathbf{n m})\end{array}$ & $\begin{array}{l}\text { Zeta } \\
\text { potential } \\
(\mathbf{m V})\end{array}$ & PDI & DLC (\%) \\
\hline PSH-AuNPs-10 & $8.2 \pm 1.5$ & $-12.3 \pm 0.4$ & $0.174 \pm 0.029$ & - \\
PSH-AuNPs-25 & $20.7 \pm 2.3$ & $-9.2 \pm 0.7$ & $0.238 \pm 0.042$ & - \\
PSH-AuNPs-50 & $41.9 \pm 4.8$ & $-5.1 \pm 1.3$ & $0.185 \pm 0.036$ & - \\
HCPT-AuNPs-10 & $9.6 \pm 1.2$ & $-4.3 \pm 1.5$ & $0.254 \pm 0.018$ & $11.28 \pm 1.38$ \\
HCPT-AuNPs-25 & $25.4 \pm 3.5$ & $-3.2 \pm 0.8$ & $0.196 \pm 0.037$ & $11.07 \pm 1.94$ \\
HCPT-AuNPs-50 & $49.2 \pm 7.8$ & $-7.9 \pm 1.6$ & $0.225 \pm 0.029$ & $9.84 \pm 1.59$ \\
\hline
\end{tabular}

Note: HCPT-AuNPs-10, 25 and 50: HCPT-AuNPs of an average diameter of 10, 25 and $50 \mathrm{~nm}$.

Abbreviations: PSH, $\alpha$-methoxy-poly (ethylene glycol)- $\omega$-mercapto; AuNPs, gold nanoparticles; HCPT, I0-hydroxycamptothecin; SD, standard deviation; PDI, polydispersity index; DLC, drug loading capacity. likely due to a structural transformation associated with the $\mathrm{pH}$ change. Under acidic conditions $(\mathrm{pH}<4)$, HCPT exists primarily as the lactone. At physiological $\mathrm{pH}(\mathrm{pH} 7.4), \sim 90 \%$ of the HCPT is in the carboxylate form. ${ }^{20}$ Furthermore, as shown in Figure 2B, the size variation of the three treatment groups was $<10 \mathrm{~nm}$ over a period of up to 4 days, indicating that HCPT-AuNPs-50 have good stability. The results suggested that the PEG layer and HCPT conjugation effectively insulated the AuNPs from nonspecific binding to proteins and that they thus displayed satisfactory serum stability. Stability studies on HCPT-AuNPs-10, HCPT-AuNPs-25, and PSHAuNPs were also carried out. All of the nano-conjugates had high stability, similar to that of HCPT-AuNPs-50.

\section{Drug adsorption and release behavior in vitro}

The HCPT adsorption curve depicted in Figure 3A illustrates that the interaction between HCPT and AuNPs was complete after preparation overnight. The in vitro release profile was obtained by measuring the percentage of drug released with respect to the total amount of HCPT encapsulated in 
A

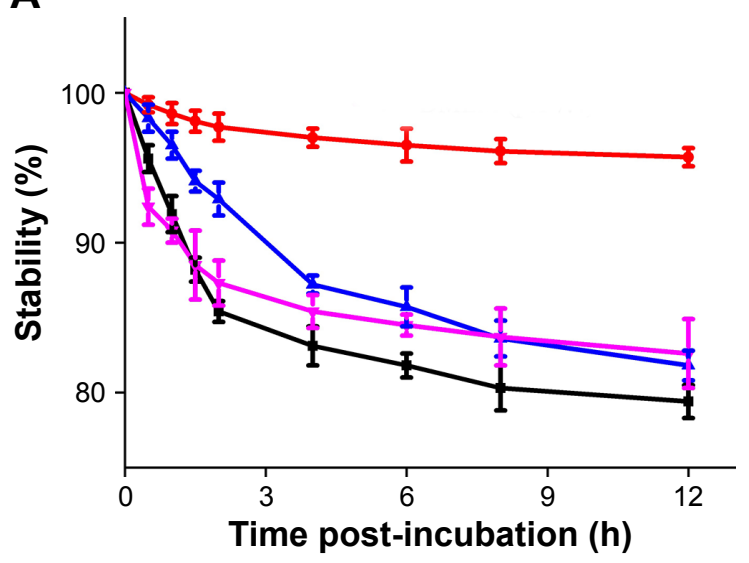

B

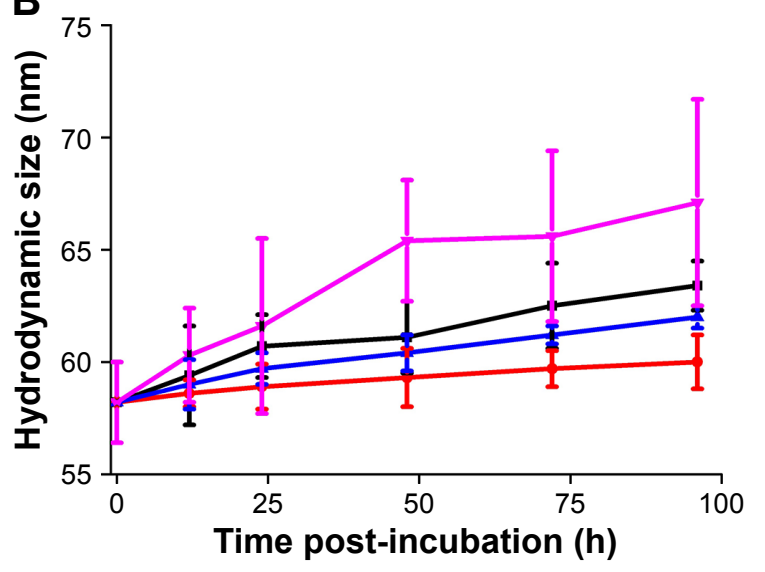

$\longrightarrow$ PBS $(\mathrm{pH} 7.4) \longrightarrow \mathrm{PBS}(\mathrm{pH} 5.5)$

PBS $(\mathrm{pH} 7.4)$ with $2 \%$ serum $\longrightarrow$ DMEM $(\mathrm{pH} 7.4)$ with $10 \%$ FBS

Figure 2 Stability analyses of HCPT-AuNPs- 50 conjugates as a function of time in different environments.

Notes: (A) Dispersion stability of nano-conjugate. (B) Size variation of nano-conjugate. HCPT-AuNPs-10, 25 and 50: HCPT-AuNPs of an average diameter of I0, 25 and $50 \mathrm{~nm}$.

Abbreviations: HCPT, 10-hydroxycamptothecin; AuNPs, gold nanoparticles; PBS, phosphate-buffered saline; DMEM, Dulbecco's Modified Eagle's Medium; FBS, fetal bovine serum.
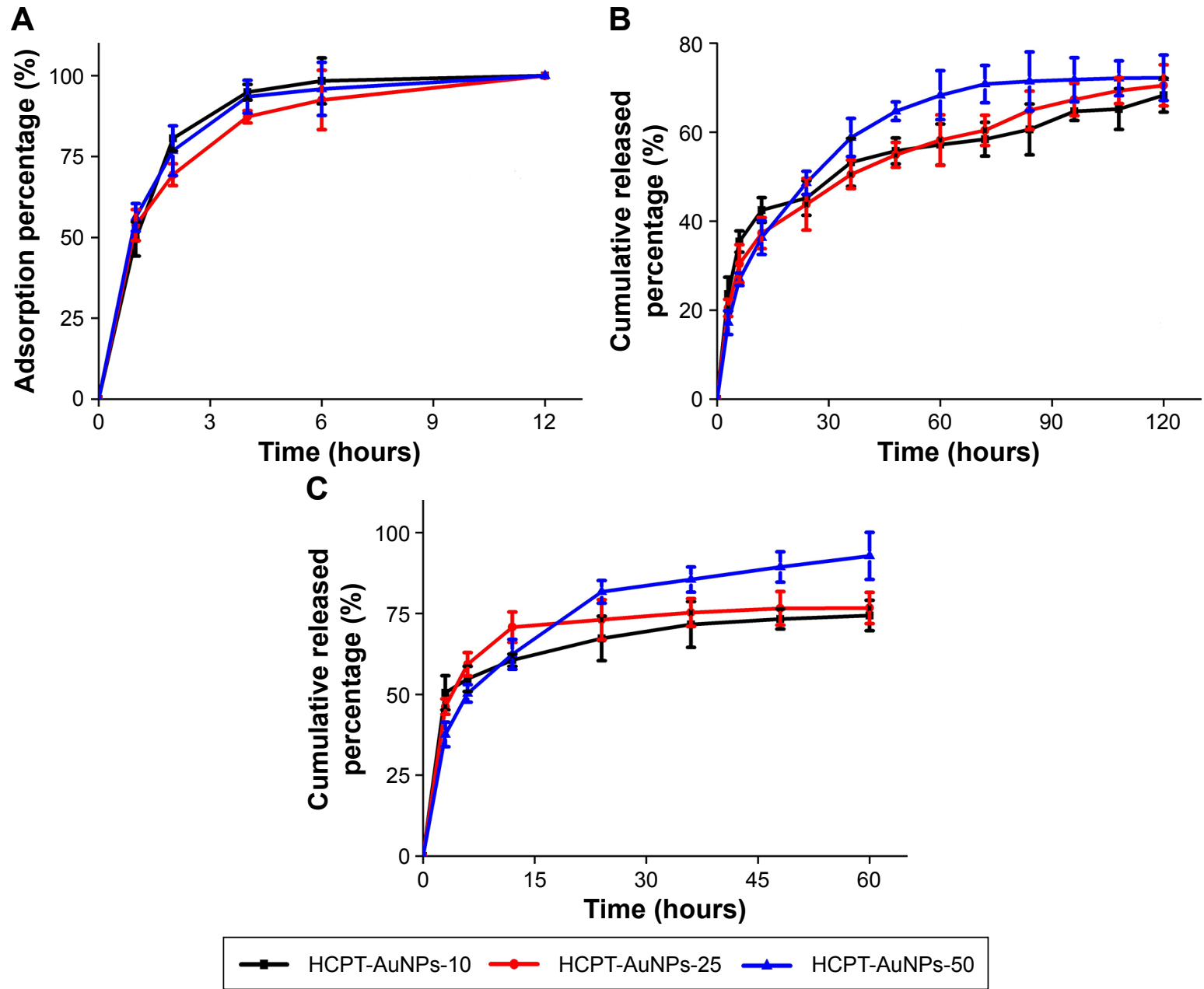

Figure 3 HCPT adsorption onto and release from AuNPs.

Notes: (A) Adsorption curve of HCPT onto HCPT-AuNPs conjugate. (B) The in vitro release of HCPT from HCPT-AuNPs conjugate in PBS. (C) The in vitro release of HCPT from HCPT-AuNPs conjugate in cell culture medium at $37^{\circ} \mathrm{C} \pm 0.5^{\circ} \mathrm{C}$. HCPT-AuNPs- 10,25 and 50 : HCPT-AuNPs of an average diameter of 10,25 and 50 nm. Abbreviations: HCPT, I0-hydroxycamptothecin; AuNPs, gold nanoparticles; PBS, phosphate-buffered saline. 
the AuNPs. The release behavior of HCPT was determined at $37^{\circ} \mathrm{C} \pm 0.5^{\circ} \mathrm{C}$ in culture medium or in PBS containing Tween $80(0.1 \%, \mathrm{w} / \mathrm{v})$ to mimic biological and nonbiological conditions, respectively. The release rate of HCPT from the HCPT-AuNPs conjugate in culture medium was faster than that in PBS (Figure 3B and C). These results can be attributed to the complicated composition of the culture medium that contains substances such as bovine serum albumin, vitamins, and amino acids, which contain carboxylic or thiol functional groups, as distinct from the composition of PBS., ${ }^{4}$ Also, we evaluated the release kinetics of HCPT for different-sized HCPT-AuNPs. All particle formulations exhibited a biphasic release profile, which was characterized by a rapid release period followed by a slower release period. Taking HCPT-AuNPs-50 as an example, $\sim 65 \%$ of HCPT is released into the culture medium and PBS after 12 and 48 hours, respectively. After the fast release stage, HCPT was released continuously for 60 and 120 hours, reaching a percentage of cumulative release of up to $92.8 \% \pm 7.3 \%$ and $72.2 \% \pm 5.1 \%$ into culture medium and PBS, respectively. Further, the HCPT nano-conjugate induced a high drug release percentage of smaller sized NPs in the first 3 hours, particularly with those of a $10 \mathrm{~nm}$ diameter, which may be due to enhanced drug loading on the surface of the AuNPs.

\section{Cellular uptake}

MDA-MB-231 cells were treated with free HCPT or different-sized HCPT-AuNPs, and the uptake of HCPT into the cells was measured. The cells were exposed to two concentrations (100 and $200 \mu \mathrm{M})$ of either HCPT or HCPTAuNPs for 4 hours (Figure 4). NPs treatment induced a significantly higher drug accumulation in the cells that was particularly obvious for HCPT-AuNPs-10. At HCPT concentrations of 100 and $200 \mu \mathrm{M}$, the amount of intracellular HCPT was four- and fivefold higher in the HCPT-AuNPs-10 group than in the free HCPT-treated group, respectively. Further, HCPT-AuNPs-25 accumulated at higher intracellular concentrations after 4-hour incubation compared with HCPT-AuNPs-50. Our investigation of NPs uptake by cells is similar to that observed with poly(lactic-co-glycolic acid) or other polymer NPs, and is a size- and concentrationdependent endocytic process. ${ }^{32,33}$ The smaller particles had a higher cellular internalization efficiency. ${ }^{34}$

\section{In vitro cytotoxicity study}

It has been reported that the cellular uptake of NPs ranging from 2 to $100 \mathrm{~nm}$ depends on their size. ${ }^{7,35}$ However, no detailed information about the uptake of different-sized

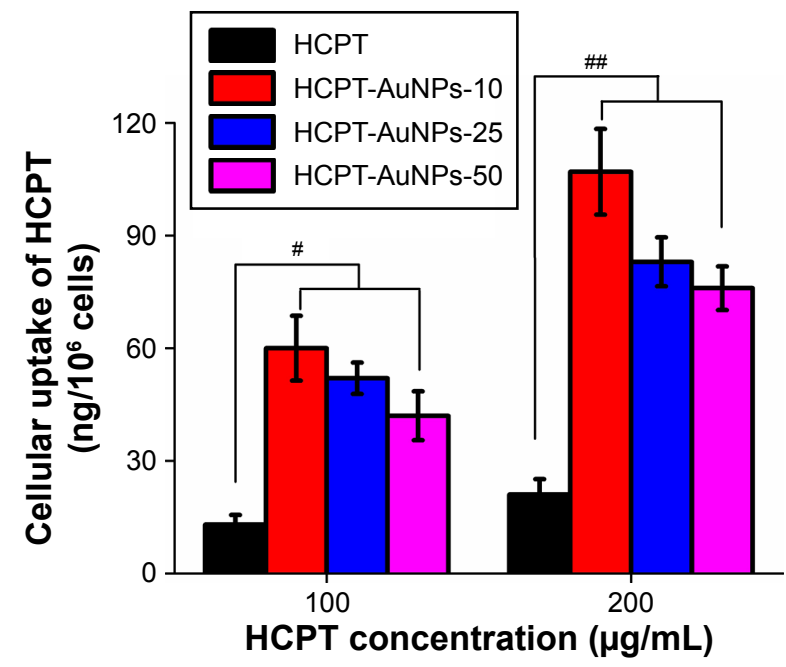

Figure 4 Uptake of HCPT-AuNPs and HCPT into MDA-MB-23I cells. Notes: Results are expressed as mean \pm SD. ${ }^{*} P<0.05$ and ${ }^{\# P} P<0.01$ compared with free HCPT. HCPT-AuNPs-10, 25 and 50: HCPT-AuNPs of an average diameter of I0, 25 and $50 \mathrm{~nm}$.

Abbreviations: HCPT, 10-hydroxycamptothecin; AuNPs, gold nanoparticles; SD, standard deviation; PBS, phosphate-buffered saline.

drug-loaded AuNPs exists. We first evaluated the cytotoxicity of HCPT-AuNPs ranging from 10 to $50 \mathrm{~nm}$ in a monolayer of MDA-MB-231 cells.

The inhibitory effects of free HCPT, PSH-AuNPs, and their conjugate HCPT-AuNPs were examined in MDA-MB231 cells using the MTT assay after 48 hours of incubation. No significant differences were seen among PSH-AuNPs of different sizes. As depicted in Figure 5A, the cytotoxicity of PSH-AuNPs on MDA-MB-231 cells at the concentration used for HCPT-AuNPs did not change with respect to the control group, suggesting that the AuNPs may be a suitable vehicle for the delivery of HCPT into cancer cells. Both the HCPT solution and HCPT nano-conjugates can significantly inhibit MDA-MB-231 cell proliferation $(P<0.05)$. Compared with the HCPT solution, a significant increase in the inhibition rate was evident in the HCPT nano-conjugates $(P<0.05)$. HCPT-AuNPs-50 had the highest cell inhibition effect among all the groups tested. The results were consistent with previous research that demonstrated that $50 \mathrm{~nm}$ citrate-stabilized AuNPs were taken up at a higher concentration than NPs of other sizes (15-100 nm). ${ }^{36}$ However, no significant difference was evident between HCPT-AuNPs-10 and HCPTAuNPs-25. Furthermore, the HCPT solution and the HCPT nano-conjugates induced cytotoxicity in MDA-MB-231 cells in a concentration-dependent manner, as depicted in Figure $5 \mathrm{~B}$. The $\mathrm{IC}_{50}$ values of the nano-conjugates were much lower than that of the free HCPT solution. Taking HCPTAuNPs-50 as an example, after 48-hour incubation, exposure to 50 and $100 \mu \mathrm{g} / \mathrm{mL}$ of the HCPT nano-conjugates inhibited cell growth by $26.6 \% \pm 4.5 \%$ and $19.7 \% \pm 2.5 \%$, whereas in 
A

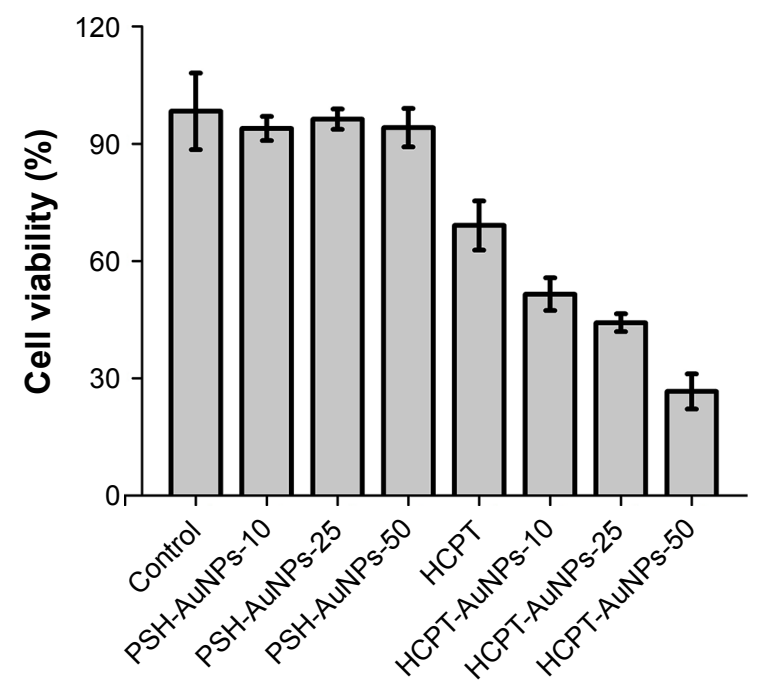

B

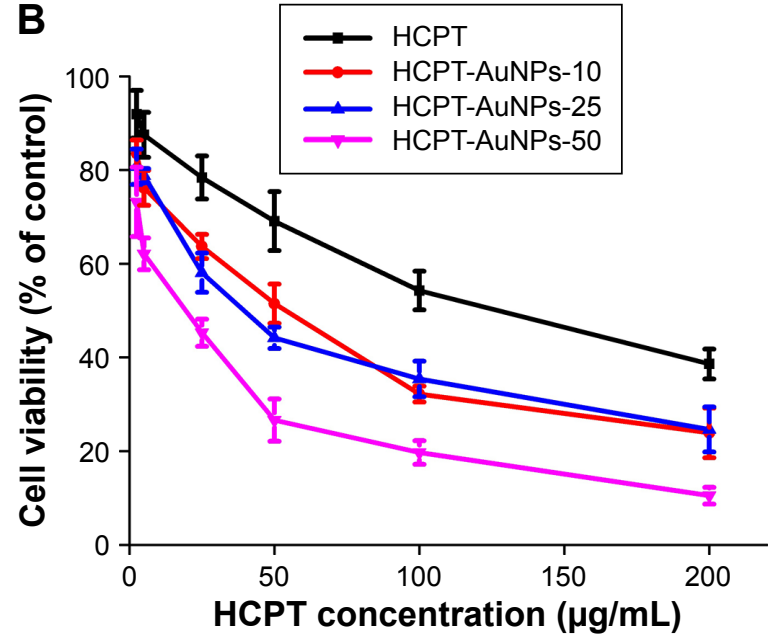

Figure $\mathbf{5}$ Cytotoxicity studies assayed by the MTT method.

Notes: (A) The influence of HCPT, different-sized PSH-AuNPs, and HCPT-AuNPs on the survival of MDA-MB-23I cells (HCPT concentration $50 \mu g / m L)$. (B) Cytotoxicity studies of different concentrations of HCPT-AuNPs and HCPT on MDA-MB-23I cells. The results are expressed as mean \pm SD. HCPT-AuNPs- 10,25 and 50 : HCPT-AuNPs of an average diameter of 10,25 and $50 \mathrm{~nm}$.

Abbreviations: MTT, 3-(4,5-dimethylthiazol-2-yl)-2,5-diphenyltetrazolium bromide; HCPT, I0-hydroxycamptothecin; PSH, $\alpha$-methoxy-poly (ethylene glycol)- $\omega$-mercapto; AuNPs, gold nanoparticles; SD, standard deviation.

free HCPT solution, it was $69.1 \% \pm 6.3 \%$ and $50.3 \% \pm 4.1 \%$, respectively. These results can be attributed to the increased solubility of HCPT by nano-conjugation. Moreover, based on the drug release measured in vitro, after 48-hour incubation, we noticed that $\sim 73 \%, 77 \%$, and $90 \%$ of HCPT was released from HCPT-AuNPs-10, HCPT-AuNPs-25, and HCPT-AuNPs-50, respectively, indicating that NPs size is a key factor that strongly impacts the drug release profile. We believe that the size is responsible for this enhanced cytotoxic effect.

It is well known that in addition to the size, the cellular uptake of NPs depends on many other factors, including the surface charge, ${ }^{37}$ the sedimentation and diffusion velocity effects of large and dense particles, ${ }^{38}$ the composition of the protein corona on the NPs, ${ }^{39}$ competition between receptors and ligands, ${ }^{5}$ and the stage of the cell cycle. ${ }^{40}$ The precise cause for the marked differences in the cytotoxicity among these three different-sized AuNPs is not clear and warrants further study.

\section{In vivo anticancer effects in MDA- MB-23I-bearing mice}

We tested the in vivo antitumor efficiency of HCPT-AuNPs by ip injection into mice with subcutaneous MDA-MB-231 tumors. The median tumor size for each group as a function of time is depicted in Figure 6C and D. The growth rate of the tumors in mice treated with blank PSH-AuNPs was indistinguishable from that in the control group, indicating that the PSH-AuNPs alone had no effect on tumor growth. The group treated with free HCPT injections showed a significantly smaller tumor volume than the control group $(P<0.05)$. The data provided strong evidence that the HCPT nano-conjugates had increased antitumor activity compared with HCPT alone. For example, the HCPTAuNPs-25 significantly outperformed the HCPT group in this study, showing a statistically significant difference from day 5 to day $21(P<0.05)$. The tumor volumes of these two groups 21 days post-drug administration were $648 \pm 87$ and $905 \pm 104 \mathrm{~mm}^{3}$ (mean \pm SD), respectively. These data confirmed the therapeutic effect of the HCPT nanoconjugates. HCPT injections formulated in the sodium salt of the carboxylate had only $10 \%$ of the cytotoxic activity of the lactone form. ${ }^{19}$ HCPT existed as an active lactone form in a larger percentage in the nano-conjugates, and the cellular uptake of this form might be enhanced by using HCPT-AuNPs. Several studies have reported that the active lactone form in the nanosuspensions was protected from conversion to the less potent carboxylate form in blood. ${ }^{18,41}$ The HCPT delivery system we constructed might also effectively protect the lactone form and consequently improve the activity of HCPT. Additionally, the enhanced permeability and retention effects led to preferential accumulation of HCPT-AuNPs in tumors. ${ }^{9}$ The sustained release of HCPT from the drug delivery system might provide a 
A

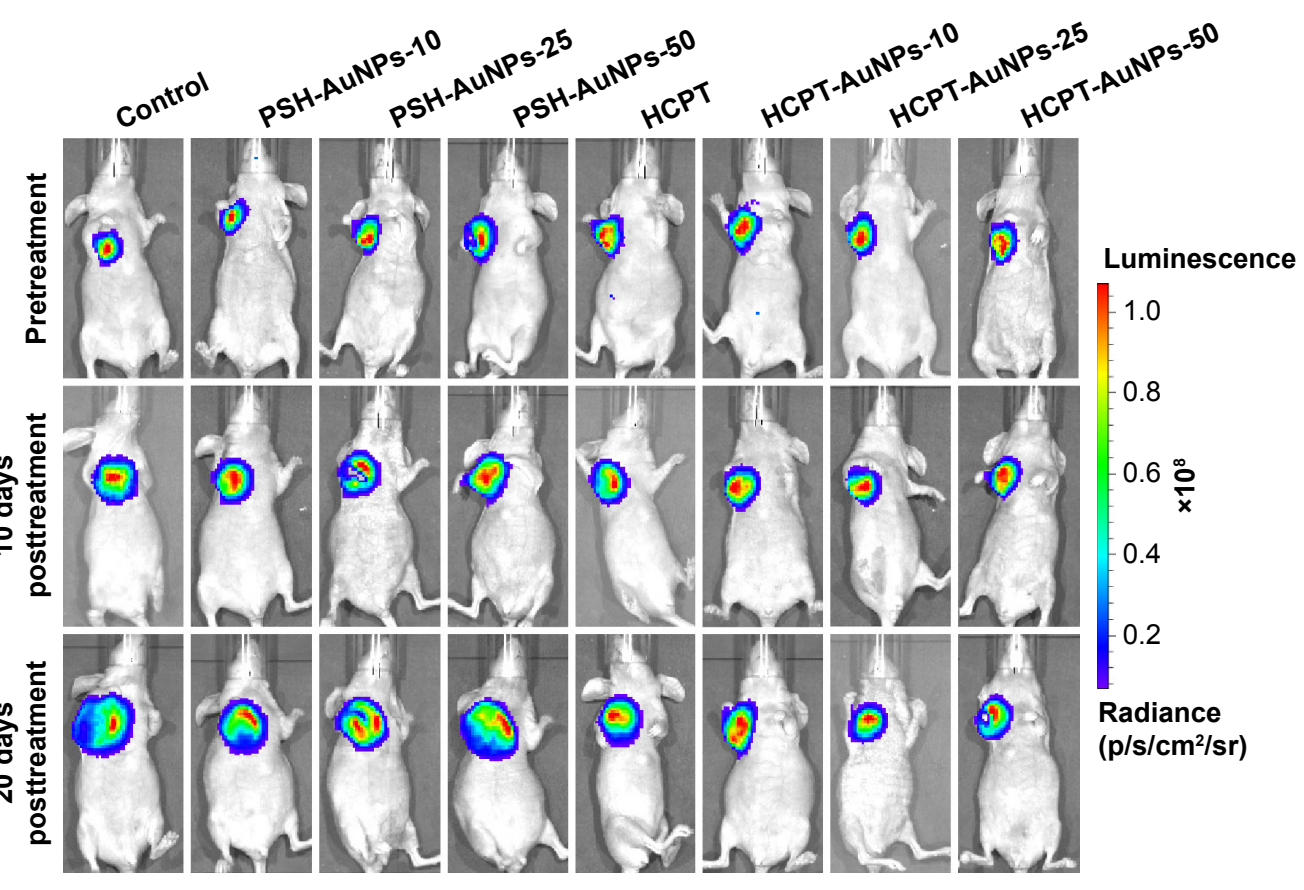

B

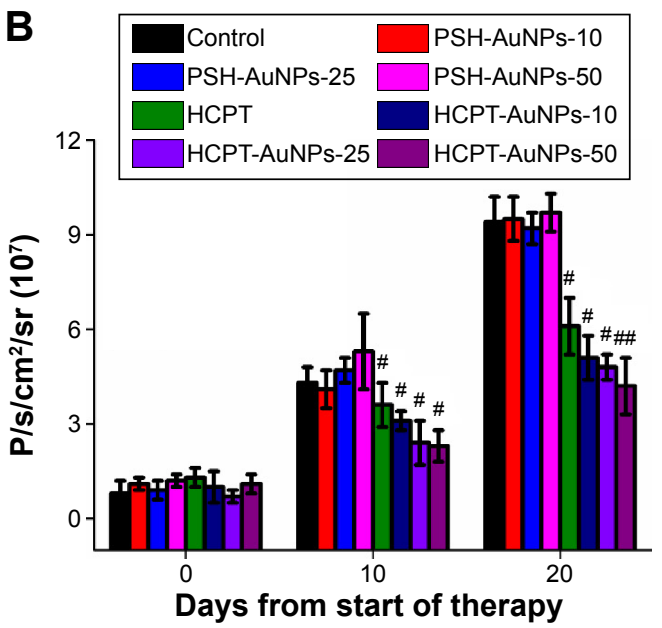

D

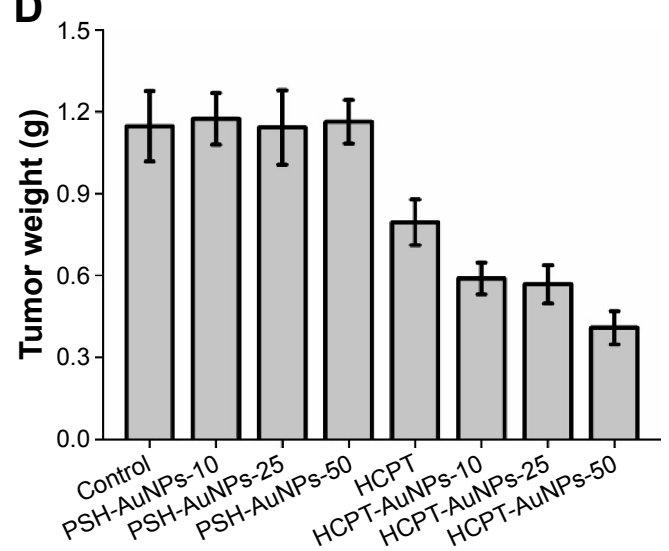

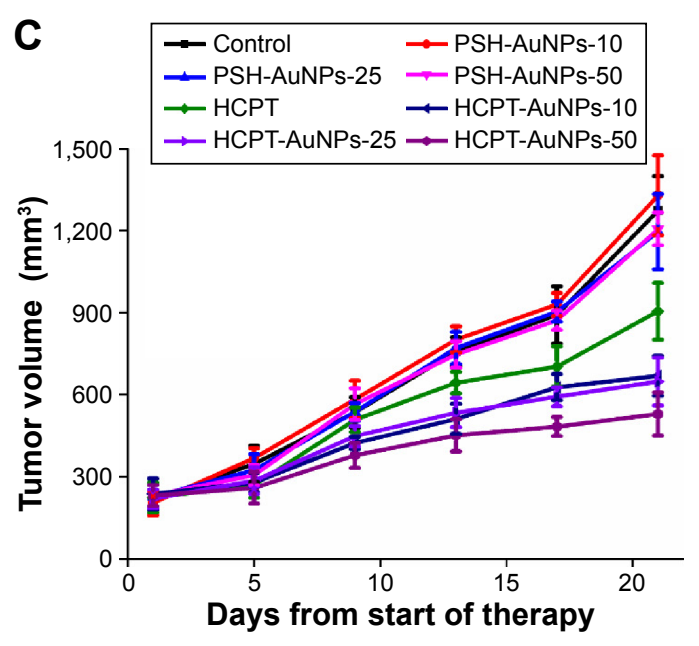

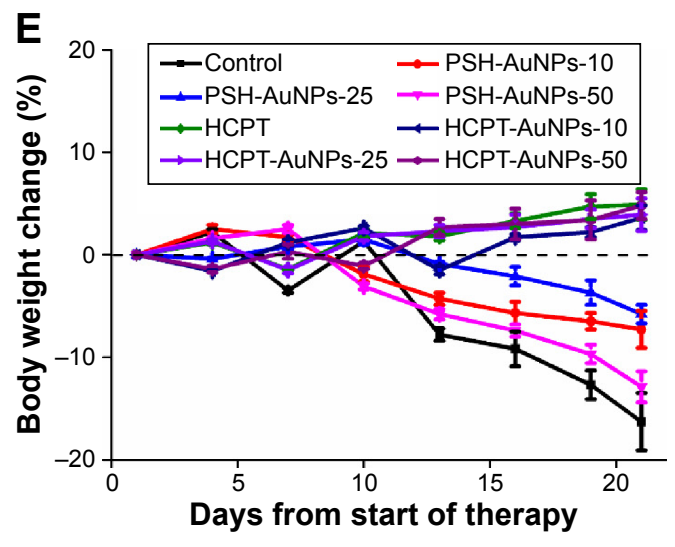

Figure 6 Antitumor effect of different sizes of PSH-AuNPs, HCPT-AuNPs, free HCPT, and a control in a xenograft model of MDA-MB-23I subcutaneous breast cancer implanted into nude mice.

Notes: (A) Bioluminescent imaging by luciferase. (B) Quantification analysis of bioluminescent imaging. ${ }^{P}<0.05$ and ${ }^{\# P<0.0 I}$ vs 0 day as control. (C) Tumor volume changes. (D) Tumor tissue weight 21 days post-therapy. (E) Body weight changes. Data were represented as mean \pm SD ( $n=5$ ). HCPT-AuNPs-I0, 25 and 50: HCPT-AuNPs of an average diameter of 10,25 and $50 \mathrm{~nm}$

Abbreviations: PSH, $\alpha$-methoxy-poly (ethylene glycol)- $\omega$-mercapto; AuNPs, gold nanoparticles; HCPT, I0-hydroxycamptothecin; SD, standard deviation. 
relatively high drug concentration in the tumor tissue. It was supposed that the drug levels in the site could reach and be maintained in the therapeutic range, resulting in increased antitumor efficiency.

In this study, we first investigated whether variations in the size of the NPs would affect the efficacy of HCPTAuNPs in an MDA-MB-231 breast xenograft model. It is noteworthy that the HCPT-AuNPs-50 group showed the best antitumor efficiency among all of the groups. The tumor volume of the HCPT-AuNPs-50 group 21 days postdrug administration was $529 \pm 79 \mathrm{~mm}^{3}$ (mean $\pm \mathrm{SD}$ ), which was smaller than in any other group $(P<0.05)$. Compared with the control group, injection of HCPT-AuNPs-50 started to show a statistically significant efficacy in delaying tumor growth beginning at day $5(P<0.05$ at days 9 and 13) and an even higher significant inhibition effect at days 17 and $21(P<0.01$ at days 17 and 21). Furthermore, the HCPT-AuNPs-50 group also significantly outperformed HCPT in this study, with a statistically significant difference present from day 5 to day $21(P<0.05)$. Of the three different-sized drug-loaded NPs, the $50 \mathrm{~nm}$ NPs had the highest antitumor efficiency, which was consistent with the in vitro studies described earlier. However, no statistically significant differences were present between the HCPTAuNPs-10 group and the HCPT-AuNPs-25 group. Size is an important parameter in a biological environment. One study has shown that NPs with diameters $\sim 100 \mathrm{~nm}$ were usually distributed around tumor blood vessels, with little penetration into the tumor parenchyma. ${ }^{42}$ Previous work demonstrated that among drug-loaded polymeric micelles with diameters ranging from 30 to $100 \mathrm{~nm}$, micelles $\sim 30 \mathrm{~nm}$ in diameter could penetrate pancreatic tumors and achieve the best antitumor effect. ${ }^{11}$ Studies on the precise mechanisms are in progress and will be presented in a subsequent report.

\section{Bioluminescence imaging of breast cancer growth in xenograft models}

We also observed the tumor status in xenografts using a bioluminescence imaging assay. Because MDA-MB-231luc cells implanted into mice emit photons in the presence of D-luciferin, the quantity of emitted photons measured via bioluminescent imaging precisely represents the tumor size in a living animal. Differences in the quantity of photons between the PSH-AuNPs and the control groups were not significant $(P>0.05)$. Mice treated with HCPT or HCPT-AuNPs all showed a significant delay in tumor growth, compared with the control group (Figure 6A and B). In addition, the quantity of photons in HCPT-AuNPs-50 was the least among three different-sized HCPT-AuNPs. These data further confirm a stronger antitumor effect of HCPT-AuNPs as compared to HCPT, and further support the more potent effect in the killing of breast cancer cells of HCPT-AuNPs with diameters of $50 \mathrm{~nm}$ than those of 10 or $25 \mathrm{~nm}$.

\section{Tolerability}

Pan et $\mathrm{al}^{43}$ demonstrated that the cytotoxicity of TPPMS/ TPPTS-modified AuNPs depended primarily on their size and not on their ligand chemistry. They observed that AuNPs of 1-2 nm in size were highly toxic, and both smaller AuNPs and larger $15 \mathrm{~nm}$ gold colloids were comparatively nontoxic. Cho et al found that $13 \mathrm{~nm}$ PEG-coated AuNPs induced acute inflammation and apoptosis in the liver. ${ }^{44}$ These toxicity findings for PEG-coated AuNPs may have important clinical implications because PEG-coated AuNPs are widely used in biomedical applications. We evaluated the physical status of the mice by observing their appetite, fur, and behavior and found no significant difference among the six groups. Animal body weight measurement demonstrated that no obvious body weight loss was present for groups treated with different-sized HCPT-AuNPs and free HCPT throughout the study (Figure 6E). For comparison, at the end of the study, the control group and PSH-AuNPs group experienced $\sim 16.3 \%$ and $\sim 12.9 \%$ body weight loss, respectively. There were no disorders of the heart, liver, spleen, lung, or kidney among the groups. Representative hematoxylin and eosin staining photomicrographs are shown in Figure 7. No overt, concomitant signs of toxicity were observed.

\section{Conclusion}

Our work provides new insights into the function of differentsized HCPT-loaded AuNPs, ranging from 10 to $50 \mathrm{~nm}$. The NPs showed a steady and sustained release profile in vitro up to 120 hours. The $50 \mathrm{~nm}$ HCPT-loaded AuNPs had unique advantages over smaller NPs in terms of killing MDA-MB231 cells and therapeutic performance in a tumor xenografted mouse model. In conclusion, this drug delivery system is an effective strategy for achieving the improvement of the therapeutic efficacy of anticancer drugs. Furthermore, our results suggest that drug-loaded AuNPs can be designed and functionalized to achieve improved antitumor efficacy by optimizing the particle size. 

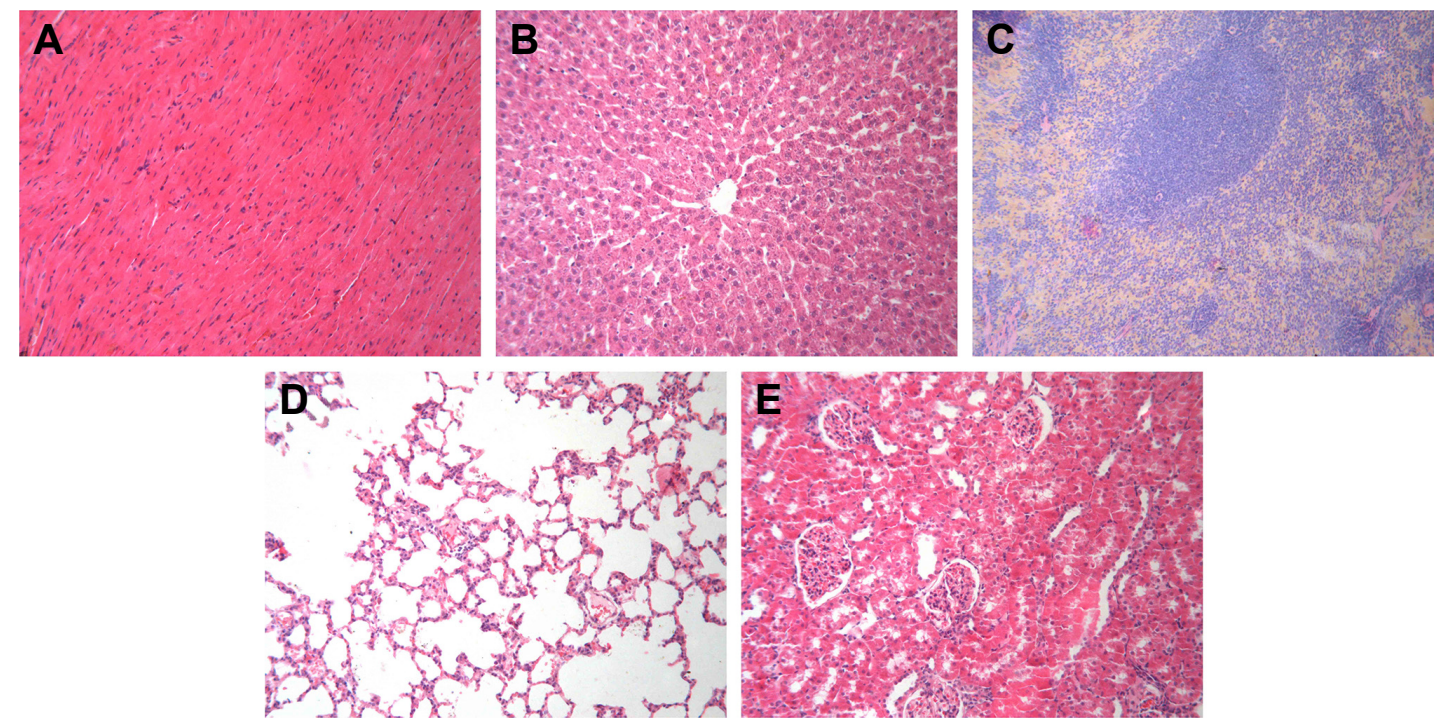

Figure 7 Histological assessment.

Notes: Representative H\&E staining photomicrographs of the (A) heart, (B) liver, (C) spleen, (D) lung, and (E) kidney in HCPT-AuNPs-50 group ( $\times 100)$. HCPT-AuNPs-50: HCPT-AuNPs of an average diameter of $50 \mathrm{~nm}$.

Abbreviations: H\&E, hematoxylin and eosin, HCPT, 10-hydroxycamptothecin; AuNPs, gold nanoparticles.

\section{Acknowledgments}

We thank Prof Ruifang Niu (Tianjin Medical University Cancer Institute and Hospital) for the gift of the MDA-MB-231-luc cells. This work was supported by National Science and Technology Major Project (No. 2013ZX09303001) and Tianjin Medical University Cancer Institute and Hospital fund (No. B1205).

\section{Author contributions}

Zhao Yan and Hanmei Bao designed the research. Hanmei Bao, Qing Zhang, and Hui Xu undertook the research. Hanmei Bao and Qing Zhang analyzed the data. Zhao Yan and Hanmei Bao wrote the paper. All authors contributed toward data analysis, drafting and critically revising the paper and agree to be accountable for all aspects of the work.

\section{Disclosure}

The authors report no conflicts of interest in this work.

\section{References}

1. Duncan B, Kim C, Rotello VM. Gold nanoparticle platforms as drug and biomacromolecule delivery systems. Journal of Controlled Release. 2010;148(1):122-127.

2. Brown SD, Nativo P, Smith JA, et al. Gold nanoparticles for the improved anticancer drug delivery of the active component of oxaliplatin. Journal of the American Chemical Society. 2010;132(13):4678-4684.

3. Setua S, Ouberai M, Piccirillo SG, Watts C, Welland M. Cisplatintethered gold nanospheres for multimodal chemo-radiotherapy of glioblastoma. Nanoscale. 2014;6(18):10865-10873.

4. Ding Y, Zhou YY, Chen H, et al. The performance of thiol-terminated PEG-paclitaxel-conjugated gold nanoparticles. Biomaterials. 2013; 34(38):10217-10227.
5. Kumar A, Ma H, Zhang X, et al. Gold nanoparticles functionalized with therapeutic and targeted peptides for cancer treatment. Biomaterials. 2012;33(4):1180-1189.

6. Arvizo RR, Bhattacharyya S, Kudgus RA, Giri K, Bhattacharya R, Mukherjee P. Intrinsic therapeutic applications of noble metal nanoparticles: past, present and future. Chemical Society Reviews. 2012;41(7): 2943-2970.

7. Jiang W, Kim BY, Rutka JT, Chan WC. Nanoparticle-mediated cellular response is size-dependent. Nature Nanotechnology. 2008;3(3): $145-150$.

8. Khlebtsov N, Dykman L. Biodistribution and toxicity of engineered gold nanoparticles: a review of in vitro and in vivo studies. Chemical Society Reviews. 2011;40(3):1647-1671.

9. Decuzzi P, Godin B, Tanaka T, et al. Size and shape effects in the biodistribution of intravascularly injected particles. Journal of Controlled Release. 2010;141(3):320-327.

10. De Jong WH, Hagens WI, Krystek P, Burger MC, Sips AJ, Geertsma RE. Particle size-dependent organ distribution of gold nanoparticles after intravenous administration. Biomaterials. 2008;29(12):1912-1919.

11. Cabral H, Matsumoto Y, Mizuno K, et al. Accumulation of sub- $100 \mathrm{~nm}$ polymeric micelles in poorly permeable tumours depends on size. Nature Nanotechnology. 2011;6(12):815-823.

12. Perrault SD, Walkey C, Jennings T, Fischer HC, Chan WC. Mediating tumor targeting efficiency of nanoparticles through design. Nano Letters. 2009;9(5):1909-1915.

13. Ruan S, He Q, Gao H. Matrix metalloproteinase triggered sizeshrinkable gelatin-gold fabricated nanoparticles for tumor microenvironment sensitive penetration and diagnosis of glioma. Nanoscale. 2015;7(21):9487-9496.

14. Ruan S, Cao X, Cun X, et al. Matrix metalloproteinase-sensitive sizeshrinkable nanoparticles for deep tumor penetration and $\mathrm{pH}$ triggered doxorubicin release. Biomaterials. 2015;60:100-110.

15. Chen KJ, Tang L, Garcia MA, et al. The therapeutic efficacy of camptothecin-encapsulated supramolecular nanoparticles. Biomaterials. 2012;33(4):1162-1169.

16. Takimoto $\mathrm{CH}$, Wright J, Arbuck SG. Clinical applications of the camptothecins. Biochimica et Biophysica Acta. 1998;1400(1-3):107-119.

17. Han R. Highlight on the studies of anticancer drugs derived from plants in China. Stem Cells (Dayton, Ohio). 1994;12(1):53-63. 
18. Pu X, Sun J, Wang Y, et al. Development of a chemically stable 10-hydroxycamptothecin nanosuspensions. International Journal of Pharmaceutics. 2009;379(1):167-173.

19. Hertzberg RP, Caranfa MJ, Holden KG, et al. Modification of the hydroxy lactone ring of camptothecin: inhibition of mammalian topoisomerase I and biological activity. Journal of Medicinal Chemistry. 1989;32(3): 715-720.

20. Han M, Liu X, Guo Y, Wang Y, Wang X. Preparation, characterization, biodistribution and antitumor efficacy of hydroxycamptothecin nanosuspensions. International Journal of Pharmaceutics. 2013;455(1-2):85-92.

21. Greenwald RB, Choe YH, Wu D. Selective phenolic acylation of 10-hydroxycamptothecin using poly (ethylene glycol) carboxylic acid. Bioorganic \& Medicinal Chemistry Letters. 2003;13(3): 577-580.

22. Zhao YX, Gao JQ, Qiao HL, Chen HL, Liang WQ. Development and validation of a sensitive reversed-phase HPLC method to determine intracellular accumulation of hydroxycamptothecin. Journal of Pharmaceutical and Biomedical Analysis. 2006;41(3): 1007-1010.

23. Lu B, Zhang Z. Novel colon-specific microspheres with highly dispersed hydroxycamptothecin cores: their preparation, release behavior, and therapeutic efficiency against colonic cancer. Journal of Pharmaceutical Sciences. 2006;95(12):2619-2630.

24. Zhao Y, Gao J, Sun X, Chen H, Wu L, Liang W. Enhanced nuclear delivery and cytotoxic activity of hydroxycamptothecin using o/w emulsions. Journal of Pharmacy \& Pharmaceutical Sciences: A Publication of the Canadian Society for Pharmaceutical Sciences, Societe canadienne des sciences pharmaceutiques. 2007;10(1):61-70.

25. Zhou L, Li X, Chen $\mathrm{X}$, et al. In vivo antitumor and antimetastatic activities of camptothecin encapsulated with N-trimethyl chitosan in a preclinical mouse model of liver cancer. Cancer Letters. 2010;297(1): 56-64.

26. Croce AC, Bottiroli G, Supino R, Favini E, Zuco V, Zunino F. Subcellular localization of the camptothecin analogues, topotecan and gimatecan. Biochemical Pharmacology. 2004;67(6):1035-1045.

27. Xie J, Lee S, Chen X. Nanoparticle-based theranostic agents. Advanced Drug Delivery Reviews. 2010;62(11):1064-1079.

28. Chen YH, Tsai CY, Huang PY, et al. Methotrexate conjugated to gold nanoparticles inhibits tumor growth in a syngeneic lung tumor model. Molecular Pharmaceutics. 2007;4(5):713-722.

29. Mosmann T. Rapid colorimetric assay for cellular growth and survival: application to proliferation and cytotoxicity assays. Journal of Immunological Methods. 1983;65(1-2):55-63.

30. Zhang L, Yang M, Wang Q, et al. 10-Hydroxycamptothecin loaded nanoparticles: preparation and antitumor activity in mice. Journal of Controlled Release. 2007;119(2):153-162.
31. Morita T, Horikiri Y, Suzuki T, Yoshino H. Preparation of gelatin microparticles by co-lyophilization with poly(ethylene glycol): characterization and application to entrapment into biodegradable microspheres. International Journal of Pharmaceutics. 2001;219(1-2):127-137.

32. Cartiera MS, Johnson KM, Rajendran V, Caplan MJ, Saltzman WM. The uptake and intracellular fate of PLGA nanoparticles in epithelial cells. Biomaterials. 2009;30(14):2790-2798.

33. Liu J, Jiang Z, Zhang S, Saltzman WM. Poly(omega-pentadecalactoneco-butylene-co-succinate) nanoparticles as biodegradable carriers for camptothecin delivery. Biomaterials. 2009;30(29):5707-5719.

34. Qaddoumi MG, Ueda H, Yang J, Davda J, Labhasetwar V, Lee VH. The characteristics and mechanisms of uptake of PLGA nanoparticles in rabbit conjunctival epithelial cell layers. Pharmaceutical Research. 2004;21(4):641-648.

35. Huang K, Ma H, Liu J, et al. Size-dependent localization and penetration of ultrasmall gold nanoparticles in cancer cells, multicellular spheroids, and tumors in vivo. ACS Nano. 2012;6(5):4483-4493.

36. Chithrani BD, Ghazani AA, Chan WC. Determining the size and shape dependence of gold nanoparticle uptake into mammalian cells. Nano Letters. 2006;6(4):662-668.

37. Kim B, Han G, Toley BJ, Kim CK, Rotello VM, Forbes NS. Tuning payload delivery in tumour cylindroids using gold nanoparticles. Nature Nanotechnology. 2010;5(6):465-472.

38. Cho EC, Zhang Q, Xia Y. The effect of sedimentation and diffusion on cellular uptake of gold nanoparticles. Nature Nanotechnology. 2011; 6(6):385-391.

39. Cedervall T, Lynch I, Lindman S, et al. Understanding the nanoparticleprotein corona using methods to quantify exchange rates and affinities of proteins for nanoparticles. Proceedings of the National Academy of Sciences of the United States of America. 2007;104(7):2050-2055.

40. Kim JA, Aberg C, Salvati A, Dawson KA. Role of cell cycle on the cellular uptake and dilution of nanoparticles in a cell population. Nature Nanotechnology. 2012;7(1):62-68.

41. Burke TG, Mi Z. Preferential binding of the carboxylate form of camptothecin by human serum albumin. Analytical Biochemistry. 1993; 212(1):285-287.

42. Matsumura Y, Maeda H. A new concept for macromolecular therapeutics in cancer chemotherapy: mechanism of tumoritropic accumulation of proteins and the antitumor agent smancs. Cancer Research. 1986;46(12 Pt 1):6387-6392.

43. Pan Y, Leifert A, Ruau D, et al. Gold nanoparticles of diameter $1.4 \mathrm{~nm}$ trigger necrosis by oxidative stress and mitochondrial damage. Small. 2009;5(18):2067-2076.

44. Cho WS, Cho M, Jeong J, et al. Acute toxicity and pharmacokinetics of 13 nm-sized PEG-coated gold nanoparticles. Toxicology and Applied Pharmacology. 2009;236(1):16-24.
International Journal of Nanomedicine

\section{Publish your work in this journal}

The International Journal of Nanomedicine is an international, peerreviewed journal focusing on the application of nanotechnology in diagnostics, therapeutics, and drug delivery systems throughout the biomedical field. This journal is indexed on PubMed Central, MedLine, CAS, SciSearch $®$, Current Contents ${ }^{\circledR} /$ Clinical Medicine,
Dovepress

Journal Citation Reports/Science Edition, EMBase, Scopus and the Elsevier Bibliographic databases. The manuscript management system is completely online and includes a very quick and fair peer-review system, which is all easy to use. Visit http://www.dovepress.com/ testimonials.php to read real quotes from published authors. 